\title{
A holomorphic representation approach to the regularization of model field theories in coupled cluster form
}

\author{
Jouko S. Arponen ${ }^{1}$ and Raymond F. Bishop ${ }^{2}$ \\ ${ }^{1}$ Department of Theoretical Physics, University of Helsinki, Siltavuorenpenger 20 C, SF-00170 \\ Helsinki, Finland \\ ${ }^{2}$ Department of Mathematics, University of Manchester Institute of Science and Technology, \\ P.O. Box 88, Manchester M60 1QD, United Kingdom
}

Received December 1, 1990; received in revised form March 6, 1991/Accepted March 19, 1991

Summary. We explain in detail the so-called Bargmann or holomorphic representation, and apply it to the general class of single-mode bosonic field theories. Since these model field theories have no attribute of separability and are, in some sense, maximally nonlocal, they are an especially severe test of the capability of coupled cluster methods to parametrize them satisfactorily. They include the cases of anharmonic oscillators of order $2 K(K=2,3, \ldots)$, for which ordinary perturbation theory is known to diverge, and we therefore make a special study of such systems. We demonstrate for the first time for any quantum-mechanical problem with infinite Hilbert space that both the normal and extended coupled cluster methods (NCCM and ECCM) have phase spaces which rigorously exist. We analyze completely the asymptotic properties of the complete sets of the NCCM and ECCM amplitudes, either of which fully characterizes the system. It is thereby shown how the holomorphic representation can be used to regularize completely all otherwise formally divergent series that appear. We demonstrate in detail how the entire NCCM and ECCM programmes can be carried through for these systems, including the diagonalization of the classically mapped Hamilitonians in the respective classical NCCM and ECCM phase spaces.

Key words: Coupled cluster theory - Anharmonic oscillators - Model field theories - Holomorphic representation - Bargmann space - Regularization Normal coupled cluster method - Extended coupled cluster method

\section{Introduction}

As several other articles in the present volume attest (and see, e.g., Refs. $[1,2]$ for reviews), the techniques of coupled cluster (CC) theory have become widely used in both chemistry and physics. Some of their particularly attractive features are their versatility, their incorporation of systematic hierarchies of approximation schemes, and the high numerical accuracy of the results that ensue from 
even relatively low-order such implementations. Indeed, the coupled cluster method (CCM) in both its so-called normal (NCCM) and extended (ECCM) manifestations [2-5], has established itself as such a universal, high-precision tool in microscopic, ab initio quantum many-body theory, that is has become relevant to address such fundamental issues as the actual existence and convergence properties of its respective parametrizations. We stress from the outset that such questions need ultimately to be addressed for all formulations of quantum many-body theory or quantum field theory. It is precisely because of the power and universality of the NCCM and ECCM parametrizations that the present analysis is now both possible and of real interest. We remark also that we know of no comparable analysis for any of the alternative general-purpose microscopic formulations based on variational, perturbative, or other techniques.

In the present analysis, our basic mathematical tool is the so-called Bargmann or holomorphic representation [6]. This provides a means whereby a bosonic quantum field theory, defined in some appropriate Hilbert space, is mapped into an essentially classical field theory of complex functions in a certain normed space, namely the Bargmann Hilbert space. One of the very attractive features of this mapping is that we may then bring to bear on the problem all of the very powerful techniques of the theory of complex functions. With this approach in mind, we therefore seek to apply the holomorphic representation to the CC parametrizations of the simplest possible bosonic field theory that is both nontrivial in itself, and which is capable of illustrating the basic features of the more general cases. With the problem thus stated, an obvious candidate presents itself, namely the anharmonic oscillator or, more generally, the class of one-body Schrödinger problems in one dimension (1-d) with arbitrary potential $V(x)$.

It is clear that such systems are numerically most easily studied using the ordinary coordinate-space one-particle Schrödinger wave function $\psi(x)$. The motivation for using more general field theoretical methods is therefore not justified by easiness of application or by accuracy of numerical results. Nevertheless, there are many reasons to exercise the general methods against such simple models. Firstly, they provide $(0+1)$-dimensional analogues of the corresponding $(d+1)$-dimensional quantum field theories in $d$ space (and one time) dimensions. For example, the quartic anharmonic oscillator is the analogue of $\phi^{4}$-field theory; and the sinusoidal (band-theory) potential well, $V(x)=k(1-\cos x)$, is the analogue of sine-Gordon field theory. Secondly, even such very simple models as the pure quartic anharmonic oscillator, $V(x)=k x^{4}$ with $k>0$, are very interesting in their own right, since it is known that ordinary RayleighSchrödinger perturbation theory performed about the corresponding harmonic oscillator as the unperturbed system, diverges for all values of the coupling constant $k$, however small [7]. In the pioneering studies of Bender and $\mathrm{Wu}$ [7] precisely this feature is considered valuable with regard to realistic field theories where perturbation expansions also typically diverge. Indeed, the asymptotic behaviour of the high-order perturbation coefficients in the expansions of physical properties is presently qualitatively known even for many realistic field theories, including QED, and they share properties similar to the anharmonic oscillator. Thirdly, when considered as interacting field theories, models like the anharmonic oscillator are rather exotic and highly singular, in the sense of being maximally nonlocal rather than local. They therefore provide a very stringent test for $\mathrm{CC}$ methods, which have been deliberately designed to incorporate the linked-cluster properties of normal physical systems exhibiting the usual locality and separability attributes. A fourth reason for studying single-mode field 
theories is that their Bargmann representations then involve functions of only a single complex variable $z$, rather than of several variables, or, more generally, functionals of a complex-valued field $z(x)$. The corresponding mathematics is thus much more familiar and more accessible.

A fifth and final reason for our interest in such single-mode bosonic field theories as the quartic anharmonic oscillator is that this particular model has already received considerable attention in terms of $\mathrm{CC}$ techniques, although from an essentially different, albeit related, viewpoint [8]. Thus, both the NCCM and ECCM are usually implemented in practice in the so-called SUB $(n)$ truncation scheme, in which only those basic amplitudes are retained which describe the excitations of at most $n$ particles out of the chosen model state, and the remaining amplitudes describing clusters of more than $n$ particles are set to zero. Unlike in the comparable SUB( $n$ ) truncation of the configuration-interaction method (CIM), for which an increase in the truncation index $n$ is guaranteed to lead to more accurate (or, in the worst case, identical) estimates for the groundand excited-state energies, due to the well-known interlacing (or Hylleraas-Undheim) theorem, no such variational or other theorem is known to exist for either the NCCM or the ECCM.

Accordingly, there has been much interest in investigating the convergence properties of the series of $n$ th-order estimates for a given physical quantity, such as the ground-state energy, as the truncation index $n$ is increased. One of the attractions of the quartic anharmonic oscillator, for example, is that the model is sufficiently simple for numerical calculations to have been performed in SUB $(n)$ approximations for values of $n$ as high as about 30 . Unfortunately, the results of all such numerical calculations to date are somewhat inconclusive. Nevertheless, there are clear indications that after very rapid initial convergence to very accurate energy-level estimates, for all values of the anharmonic coupling constant, for $n \leqslant 8$, the rate of convergence slows down dramatically for higher vaiues of $n$, and indeed generally starts to show the oscillatory behaviour typical of asymptotic expansions.

From the above standpoint, we stress that the convergence properties of interest in the present work are not those of the SUB $(n)$ sequences for given physical quantities. Rather, we are now concerned with the behaviour of the exact amplitudes which fully parametrize the system, namely $\left\{s_{n}, \tilde{s}_{n}\right\}$ in the NCCM and $\left\{\sigma_{n}, \tilde{\sigma}_{n}\right\}$ in the ECCM, as the index $n \rightarrow \infty$. One of our main findings is that both CC parametrizations actually require us to extend the usual Hilbert space of normalizable wave functions to a more general linear vector space. (Indeed, we note that this is certainly also true in $\operatorname{SUB}(n)$ approximations for $n>2$, for which it is very easy to prove that all such approximate wave functions have infinite norms.)

An important corollary of the above is that formally divergent series for various quantities in the theory appear, when expressed in terms of the basic CC coefficients $\left\{s_{n}, \tilde{s}_{n}\right\}$ or $\left\{\sigma_{n}, \tilde{\sigma}_{n}\right\}$. Nevertheless, by the use of the holomorphic representation, we are able to show how such series can be regularized to be given precise, but generally non-unique, interpretations. It seems that the intrinsically nonlinear CC parametrizations necessarily lead to mappings of the original quantum field theory in its Hilbert space onto a corresponding multiconfigurational classical field theory, which while certainly exact, are one-tomany rather than one-to-one. Nevertheless, this subsequent non-uniqueness of the CC coefficients has no effect on any such physically observable quantity as the expectation value of an arbitrary operator. One of the important outcomes 
of our study is that it provides a very complete analysis of the asymptotic structure of all of the various CC amplitudes which fully and exactly characterize the system. It is clear that some details in the present results are rather system-specific, and reflect the fact that the present system as a field theory has no attributes of locality. The more interesting cases which must form a challenging project for the future are the theories in $d$ spatial dimensions with $d \geqslant 1$. Nevertheless, this is the first time that exact asymptotic results have been derived in any CC framework, although many other NCCM applications exist (for a review see [2]). In particular, these include several detailed numerical calculations of anharmonic oscillators [8] in various $\operatorname{SUB}(n)$ approximations. We stress again that the main aim of the present work is to examine analytically the exact asymptotic properties of the various $\mathrm{CC}$ schemes rather than to investigate the numerical accuracy of actual finite truncations.

Finally, we emphasize that the $\mathrm{CC}$ methods in their variational formulation map the quantum mechanical problem into a classical canonical Hamiltonian problem in a sympletic manifold or phase space. In particular, the canonical coordinates in the ECCM phase space are fully quasilocal, and each of them represents the sum of a well-defined infinite set of connected diagrams. In this respect the methods are distinctively different from such other previous approaches as those which are mentioned in Ref. [7]. For example, the method of Truong is based on the Weyl representation of the Schrödinger wave function, and is thus close to the coherent-state expansion. Nevertheless, such representations essentially afford a reformulation for the quantum theory and are not strictly equivalent to a quasilocal classical canonical formalism of the sort that we are presently interested in.

The outline of the remainder of the paper is as follows. In Sect. 2 we introduce the main elements of the Bargmann representation of an arbitrary state of a single-mode bosonic field theory, and in Sect. 3 we then derive the corresponding holomorphic representation of both the NCCM and ECCM parametrizations of this model. In Sect. 4 we discuss the analytic properties of the various $\mathrm{CC}$ amplitudes and their Fourier transforms, and introduce the average value functional. In Sect. 5 we consider a system which is infinitesimally displaced from the equilibrium, and use the holomorphic wave functions of the excited states, assumed to be known, to derive the forms of the various CC amplitudes. This allows us to obtain the diagonalized nonlinear expansions for the classically mapped CC Hamiltonians in their respective phase spaces. Finally, Sect. 6 summarizes our results.

\section{Coherent states and the Bargmann representation of arbitrary states}

We consider a one-body Schrödinger quantum-mechanical problem in one space dimension $x \in(-\infty, \infty)$, with Hamiltonian:

$$
H=\frac{1}{2} \hat{p}^{2}+V(\sqrt{2} \hat{x})
$$

where the factor $\sqrt{2}$ in the potential $V$ has been inserted for later convenience. The position operator $\hat{x}(\rightarrow x)$ and its canonically conjugate momentum operator $\hat{p}(\rightarrow-i d / d x)$ are conventionally mapped onto their canonical Fock-space creation and destruction counterparts by:

$$
a^{\dagger}=(\hat{x}-i \hat{p}) / \sqrt{2}, \quad a=(\hat{x}+i \hat{p}) / \sqrt{2} .
$$


They are thus easily seen to obey the usual bosonic canonical commutation relation (CCR):

$$
\left[a, a^{\dagger}\right]=I \text {. }
$$

The corresponding (normalized) vacuum state $|\phi\rangle$ defined by:

$$
a|\phi\rangle=0 \text {, }
$$

now plays the role of model state or cyclic vector. It is simply the ground state of the harmonic oscillator Hamilitonian:

$$
H^{0}=\frac{1}{2} \hat{p}^{2}+\frac{1}{2} \hat{x}^{2}=a^{\dagger} a+\frac{1}{2},
$$

and it has the usual Gaussian form for its coordinate-space representation:

$$
\langle x \mid \phi\rangle \equiv \phi(x)=\pi^{-1 / 4} \exp \left(-\frac{1}{2} x^{2}\right) ; \quad\langle\phi \mid \phi\rangle=1 .
$$

For such single-mode field theories, the complete set of orthonormal multiconfigurational creation operators with respect to $|\phi\rangle$ are simply the $n$-boson creation operators, $(n !)^{-1 / 2}\left(a^{\dagger}\right)^{n}$. From present purposes it is actually more convenient to work directly with their unnormalized counterparts, $\left(a^{\dagger}\right)^{n}$, and to use the manifest normalization condition:

$$
\langle m \mid n\rangle=\delta_{m n} ; \quad|n\rangle \equiv(n !)^{-1 / 2}\left(a^{\dagger}\right)^{n}|\phi\rangle,
$$

as needed. It is clear that arbitrary ket and bra states in the Fock space may now be represented in the CIM form as:

$$
\begin{aligned}
& |g\rangle \equiv g\left(a^{\dagger}\right)|\phi\rangle, \quad\left\langle f^{*}\right| \equiv\langle\phi| f(a)=\left[f^{*}\left(a^{\dagger}\right)|\phi\rangle\right]^{\dagger}=\left(\left|f^{*}\right\rangle\right)^{\dagger} \\
& g(z) \equiv \sum_{n=0}^{\infty} g_{n} z^{n}, \quad f(z) \equiv \sum_{n=0}^{\infty} f_{n} z^{n}
\end{aligned}
$$

where, as usual, an asterisk denotes complex conjugation.

The remaining ket eigenstates $|z\rangle$ of the operator $a$ :

$$
a|z\rangle=z|z\rangle \text {, }
$$

where $z$ is an arbitrary complex number, also play a fundamental role. They are the well-known Glauber coherent states. Their normalized forms are easily constructed from the defining relation of Eq. (9) as:

$$
|z\rangle=e^{\left(z a^{\dagger}-z^{*} a\right)}|\phi\rangle=e^{-\frac{1}{2}|z|^{2}} e^{z a^{\dagger}}|\phi\rangle,
$$

the first form of which easily manifests the normalization, $\langle z \mid z\rangle=1$, and the second form of which follows from the first by a trivial use of the Baker-Campbell-Hausdorff theorem for any two operators $A$ and $B$ which separately commute with their own commutator:

$$
e^{A+B}=e^{A} e^{B} e^{-\frac{1}{2}[A, B]} ; \quad[A,[A, B]]=0=[[A, B], B] .
$$

We thus also see that the Glauber coherent states are simply the SUB(1) class of $\mathrm{CC}$ ket states with respect to $|\phi\rangle$ as the model state. By making use of Eq. (2), and Eq. (11) for $\hat{x}$ and $\hat{p}$, it is easy to verify from Eq. (10) that the coherent states have the coordinate-space representation:

$$
\langle x \mid z\rangle=\pi^{-\frac{1}{4}} \exp \left(-\frac{1}{2}|z|^{2}-\frac{1}{2} z^{2}+\sqrt{2} x z-\frac{1}{2} x^{2}\right) .
$$


It is also quite straightforward to verify that they satisfy the well-known completeness and inner-product relations:

$$
\begin{aligned}
& I=\frac{1}{\pi} \int d^{2} z|z\rangle\left\langle z\left|=\int_{-\infty}^{\infty} d x\right| x\right\rangle\langle x|, \\
& \left\langle z \mid z^{\prime}\right\rangle=\exp \left(-\frac{1}{2}|z|^{2}-\frac{1}{2}\left|z^{\prime}\right|^{2}+z^{*} z^{\prime}\right) .
\end{aligned}
$$

Equation (13) is perhaps most easily proven by taking matrix elements between arbitrary members $|n\rangle$ and $\langle m|$ of the complete basis given in Eq. (7). We also note from Eq. (14) that the arbitrary states of Eq. (8) have the coherent-state representations:

$$
\langle z \mid g\rangle=g\left(z^{*}\right) e^{-\frac{1}{2}|z|^{2}}, \quad\left\langle f^{*} \mid z\right\rangle=f(z) e^{-\frac{1}{2}|z|^{2}} .
$$

The CCR algebra of Eq. (3) is now represented in the Bargmann space by the algebra:

$$
a^{\dagger} \rightarrow z, \quad a \rightarrow d / d z,
$$

of the complex variable $z$ and its derivative. Arbitrary states in the Fock space now have the equivalent holomorphic or Bargmann representations:

$$
\begin{gathered}
f(a) g\left(a^{\dagger}\right)|\phi\rangle \equiv h\left(a^{\dagger}\right)|\phi\rangle \Leftrightarrow f(d / d z) g(z)=h(z), \\
\langle\phi| f(a) g\left(a^{\dagger}\right) \equiv\langle\phi| \tilde{h}(a) \Leftrightarrow g(d / d z) f(z)=\tilde{h}(z) .
\end{gathered}
$$

The original single-mode bosonic field theory is thereby mapped into the corresponding (classical) field theory of a complex function in a particular normed space, namely the Bargmann Hilbert space [6]. Various forms for the inner product between two arbitrary states may now be constructed, amongst which we include the following:

$$
\begin{aligned}
\left\langle f^{*} \mid g\right\rangle & =\frac{1}{\pi} \int d^{2} z e^{-|z|^{2}} f(z) g\left(z^{*}\right)=\frac{1}{\pi} \int d^{2} z e^{-|z|^{2}} f\left(z^{*}\right) g(z) \\
& =\left.f(d / d z) g(z)\right|_{z=0}=\left.g(d / d z) f(z)\right|_{z=0} \\
& =\sum_{n=0}^{\infty} n ! f_{n} g_{n} .
\end{aligned}
$$

Thus, Eq. (18a) is readily proven by insertion of a complete set of coherent states from Eq. (13), and by making use of Eq. (15). Equation (18c) follows both from Eqs. (7) and (8), and also directly from Eq. (18a) by making use of the following elementary integral over the entire complex plane:

$$
\frac{1}{\pi} \int d^{2} z z^{m}\left(z^{*}\right)^{n} e^{-|z|^{2}}=n ! \delta_{m n} .
$$

Finally Eq. (18b) follows both from Eq. (17), and also from the power series expansions of Eq. (8) as another way of writing Eq. (18b).

It is now very important to realize that if the two above states $\left\langle f^{*}\right|$ and $|g\rangle$ are normalizable, then Eq. (18a) implies that both functions $f(z)$ and $g(z)$ must be holomorphic or entire functions of order $\varrho \leqslant 2$ (and of type $\tau \leqslant \frac{1}{2}$ in the limiting case $\varrho=2$ ). It is for this reason that the Bargmann representation is otherwise known as the holomorphic representation. Furthermore, in this case it 
is not difficult to show that each of the three relations of Eqs. (18a) $-(18 \mathrm{c})$ yields the same (convergent) result. On the other hand, differences can arise when the Hilbert space of normalizable wave functions is generalized to a larger linear vector space which encompasses states of infinite norm in terms of the standard metrics. As we shall later see, it is precisely such an extension which is necessitated by the CCM parametrizations of the state vectors, and which hence brings into question the meaning which may be associated with the divergent expressions which inevitably arise as a consequence.

Before turning to the $\mathrm{CC}$ parametrizations of states, we first note that the ground-state Schrödinger equation:

$$
H\left|\psi_{0}\right\rangle=E_{0}\left|\psi_{0}\right\rangle
$$

with the Hamiltonian of Eq. (1), may now be rewritten in terms of the CIM parametrization:

$$
\left|\psi_{0}\right\rangle=F^{0}\left(a^{\dagger}\right)|\phi\rangle
$$

in terms of the following ordinary differential equation in the complex plane of the Bargmann space:

$$
-\frac{1}{4}(d / d z-z)^{2} F^{0}(z)+V(d / d z+z) F^{0}(z)=E_{0} F^{0}(z) .
$$

By writing $F^{0}(z)$ in the form:

$$
F^{0}(z) \equiv e^{-\frac{1}{2} z^{2}} f^{0}(z)
$$

Eq. (22) can be equivalently written in the somewhat simpler form:

$$
-\left(\frac{1}{2} \frac{d}{d z}-z\right)^{2} f^{\circ}(z)+V\left(\frac{d}{d z}\right) f^{0}(z)=E_{0} f^{\circ}(z)
$$

Finally, we note that the relationship between the Schrödinger representation, $\psi(x) \equiv\langle x \mid \psi\rangle$, of an arbitrary wave function $|\psi\rangle$, and its holomorphic representation $F(z)$ :

$$
|\psi\rangle=F\left(a^{\dagger}\right)|\phi\rangle, \quad F(z) \equiv \sum_{n=0}^{\infty} F_{n} z^{n},
$$

is a relatively simple linear one. Thus, by considering the overlap $\left\langle z^{*} \mid \psi\right\rangle$, by inserting into it a complete set of position eigenstates as in Eq. (13), and by making use of the coordinate-space representation of the coherent states from Eq. (12), it is straightforward to derive the Fourier-like mapping:

$$
F(z)=\pi^{-\frac{1}{4}} e^{-\frac{1}{2} z^{2}} \int_{-\infty}^{\infty} d x \exp \left(\sqrt{2} z x-\frac{1}{2} x^{2}\right) \psi(x) .
$$

Similarly, by considering the Fourier transform of Eq. (26), or by likewise considering the overlap $\langle x \mid \psi\rangle$ and inserting a complete set of coherent states as in Eq. (13), one may also derive the two equivalent inverse relations to Eq. (26), namely:

$$
\begin{aligned}
\psi(x) & =2^{-\frac{1}{2}} \pi^{-\frac{3}{4}} e^{\frac{1}{2} x^{2}} \int_{-\infty}^{\infty} d \eta \exp \left(-i \sqrt{2} x \eta-\frac{1}{2} \eta^{2}\right) F(i \eta), \\
& =\pi^{-\frac{5}{4}} e^{-\frac{1}{2} x^{2}} \int d^{2} z \exp \left(-|z|^{2}-\frac{1}{2} z^{2}+\sqrt{2} x z\right) F\left(z^{*}\right) .
\end{aligned}
$$


Having thus introduced the Bargmann representation of an arbitrary state parametrized in its CIM form, it is then natural to extend the discussion to the corresponding NCCM and ECCM parametrizations. This we now do in Sect. 3.

\section{Holomorphic representations of the $\mathrm{CC}$ operators}

In the CCM we usually choose for convenience the intermediate normalization scheme in which:

$$
\langle\phi \mid \psi\rangle=\int_{-\infty}^{\infty} d x \phi(x) \psi(x)=1,
$$

which, from Eq. (25) implies the relation:

$$
F_{0}=1 \text {. }
$$

It is also convenient to introduce the notation:

$$
\begin{aligned}
& \langle\tilde{\psi}| \equiv N^{-2}\langle\psi| \equiv\langle\phi| \tilde{F}(a) ; \quad \tilde{F}(a)=N^{-2} F^{*}(a), \\
& N^{2} \equiv\langle\psi \mid \psi\rangle=\int_{-\infty}^{\infty} d x|\psi(x)|^{2}=\left\langle\phi\left|F^{*}(a) F\left(a^{\dagger}\right)\right| \phi\right\rangle .
\end{aligned}
$$

With this normalization, the usual CCM representation of the ket wave function $|\psi\rangle$ is given as:

$$
|\psi\rangle \equiv e^{S\left(a^{\dagger}\right)}|\phi\rangle, \quad S(z) \equiv \sum_{n=1}^{\infty} s_{n} z^{n}
$$

in terms of a creation operator $S=S\left(a^{\dagger}\right)$, the cluster correlation operator, (which is more usually denoted as $T$ in quantum chemistry). We thus have the simple relation:

$$
S(z)=\ln F(z),
$$

which shows immediately that, unlike $F(z)$, the function $S(z)$ is not holomorphic. Instead, it is a multivalued function with branch points at each of the zeros of $F(z)$. We remind the reader that in general an entire function of fractional order has an infinite number of such zeros in the complex plane.

Furthermore, in the NCCM [2-5] the bra wave function $\langle\tilde{\psi}|$ is also parametrized independently in terms of a new destruction operator $\tilde{S}=\tilde{S}(a)$ as:

$$
\langle\tilde{\psi}| \equiv\langle\phi| \tilde{S}(a) e^{-S\left(a^{\dagger}\right)}, \quad \tilde{S}(z) \equiv \sum_{n=0}^{\infty} \tilde{s}_{n} z^{n} ; \quad \tilde{s}_{0}=1,
$$

which turns out (and see, e.g., Refs. [3-5]) to be canonically conjugate to $S$, and where the normalization $\langle\vec{\psi} \mid \psi\rangle=1$ from Eq. (29) implies the condition $\tilde{s}_{0}=1$. From Eqs. (29) -(31) we thus have immediately the relation:

$$
\langle\phi| \widetilde{S}(a)=N^{-2}\langle\phi| F^{*}(a) F\left(a^{\dagger}\right),
$$

and hence from Eq. (17) we also have the Bargmann representation of $\widetilde{S}(z)$ directly in terms of $F(z)$ as:

$$
\tilde{S}(z)=N^{-2} F(d / d z) F^{*}(z) .
$$

We may also similarly relate $\tilde{S}(z)$ to the Schrödinger wave function $\psi(x)$. 
Perhaps the simplest way is as follows. Let us first define an average value of an arbitrary operator $O=O\left(a^{\dagger}, a\right)$ as:

$$
\langle O\rangle \equiv \bar{O} \equiv\langle\tilde{\psi}|O| \psi\rangle=N^{-2}\left\langle\phi\left|F^{*}(a) O\left(a^{\dagger}, a\right) F\left(a^{\dagger}\right)\right| \phi\right\rangle .
$$

We then see immediately from Eq. (7) that the coefficients $\tilde{s}_{n}$ from Eq. (32) are given simply as:

$$
\tilde{s}_{n}=\frac{1}{n !}\left\langle\left(a^{\dagger}\right)^{n}\right\rangle,
$$

and hence that $\tilde{S}(z)$ is the moment generating function for powers of the creation operator $a^{\dagger}$ :

$$
\tilde{S}(z)=\left\langle e^{z a^{\dagger}}\right\rangle \text {. }
$$

Now, by inserting a complete set of position eigenstates from Eq. (13) into Eq. (36b), and by making use of Eq. (2), we easily find the result:

$$
\tilde{S}(z)=\frac{1}{N^{2}} \int_{--\infty}^{\infty} d x \psi^{*}(x) e^{z(x-d / d x) / \sqrt{2}} \psi(x) .
$$

A trivial use of the Baker-Campbell-Hausdorff theorem of Eq. (11) in Eq. (37), then gives the desired final expression:

$$
\tilde{S}(z)=\frac{1}{N^{2}} e^{-\frac{1}{4} z^{2}} \int_{-\infty}^{\infty} d x e^{z x / \sqrt{2}} \psi^{*}(x) \psi(x-z / \sqrt{2}) .
$$

We turn out attention next to the ECCM parametrization of ket and bra states. These are given initially in terms of the previous NCCM creation operators $S=S\left(a^{\dagger}\right)$ and a new destruction operator $\tilde{\Sigma}=\tilde{\Sigma}(a)$ as:

$$
\begin{aligned}
& |\psi\rangle \equiv e^{S\left(a^{\dagger}\right)}|\phi\rangle=e^{S\left(a^{\dagger}\right)} e^{-\tilde{\Sigma}(a)}|\psi\rangle ; \\
& \langle\tilde{\psi}|=\langle\phi| e^{\tilde{\Sigma}(a)} e^{-S\left(a^{\dagger}\right)}, \quad \tilde{\Sigma}(z) \equiv \sum_{n=1}^{\infty} \tilde{\sigma}_{n} z^{n} .
\end{aligned}
$$

A comparison of Eqs. (32), (36b) and (39) shows immediately that:

$$
\tilde{\Sigma}(z)=\ln \tilde{S}(z)=\ln \left\langle e^{z a^{\dagger}}\right\rangle,
$$

and hence the coefficients $\left\{\tilde{\sigma}_{n}\right\}$ are simply the connected averages of the powers of the creation operator $a^{\dagger}$ :

$$
\tilde{\sigma}_{n}=\frac{1}{n !}\left\langle\left(a^{\dagger}\right)^{n}\right\rangle_{\text {conn. }} .
$$

Thus, the relationship between the unlinked NCCM coefficients $\left\{\tilde{s}_{n}\right\}$ and their linked ECCM counterparts $\left\{\tilde{\sigma}_{n}\right\}$ is precisely that between the moments and the cumulants (or semi-invariants) of a probability distribution.

Finally, although the ECCM parametrization may be completely specified in terms of the linked-cluster set of coefficients $\left\{s_{n}, \tilde{\sigma}_{n}\right\}$, it has been explained elsewhere [2-5] that just as the NCCM operators are canonically conjugate to each other in a well-defined classical sense, so can we also define a new creation operator $\Sigma=\Sigma\left(a^{\dagger}\right)$ canonically conjugate to $\tilde{\Sigma}=\tilde{\Sigma}(a)$, by the definition:

$$
\Sigma\left(a^{\dagger}\right)|\phi\rangle \equiv(I-|\phi\rangle\langle\phi|) \tilde{S}(a) S\left(a^{\dagger}\right)|\phi\rangle, \quad \Sigma(z) \equiv \sum_{n=1}^{\infty} \sigma_{n} z^{n},
$$


and hence from Eq. (17) we also have the equivalent Bargmann representation:

$$
\Sigma(z)=\tilde{S}(d / d z) S(z)-\left.\tilde{S}(d / d z) S(z)\right|_{z=0} .
$$

In this way, the ECCM parametrization is now given in terms of the complete set of amplitudes $\left\{\sigma_{n}, \tilde{\sigma}_{n}\right\}$, all of which are again of linked-cluster type, and where $\sigma_{n}$ and $\tilde{\sigma}_{n}$ are canonically conjugate classical variables. Alternative expressions for $\Sigma(z)$ and its coefficients $\left\{\sigma_{n}\right\}$ are easily derived from Eq. (41), namely:

$$
\begin{aligned}
\sigma_{n} & =\frac{1}{n !}\left\langle\phi\left|\tilde{S}(a) a^{n} S\left(a^{\dagger}\right)\right| \phi\right\rangle=\frac{1}{n !} \sum_{m=0}^{\infty}(m+n) ! \tilde{s}_{m} s_{m+n}, \\
\Sigma(z) & =\left\langle\phi\left|\tilde{S}(a)\left(e^{z a}-1\right) S\left(a^{\dagger}\right)\right| \phi\right\rangle .
\end{aligned}
$$

It is clear once again that, like $S(z)$, the function $\Sigma(z)$ is not necessarily an entire function.

We note at this point that in general, due to the analytic properties of the complex functions $S(z)$ and $\tilde{S}(z)$, neither the ket state $S\left(a^{\dagger}\right)|\phi\rangle$ nor the bra state $\langle\phi| S(a)$ is normalizable within the original Hilbert space. In turn, expressions like those in Eq. (42) therefore have to be handled with extreme care. For example, it is generally the case that the infinite sum in Eq. (42) which expresses the ECCM coefficients $\left\{\sigma_{n}\right\}$ in terms of their NCCM counterparts $\left\{s_{n}, \tilde{s}_{n}\right\}$, is formally divergent. It is one of the purposes of the remainder of this paper to show how meaning can be given to such expressions. As we shall see, such divergences typically arise from the incorrect expansion of certain otherwise exact (convergent) integrals. In this sense the Bargmann-space representation techniques provide a definite regularization of these various divergent expressions.

Before proceeding with the above programme, we end this Section with a discussion of the Bargmann-space representation of the expectation value $\bar{O}$ of an arbitrary operator $O$ as given in Eq. (35). Firstly, in the CIM parametrization we clearly have:

$$
\begin{aligned}
\bar{O} & =N^{-2}\left\langle\phi\left|F^{*}(a) O\left(a^{\dagger}, a\right) F(a)\right| \phi\right\rangle \\
& =\left.F^{*}(d / d z) O(z, d / d z) F(z)\right|_{z=0} /\left.F^{*}(d / d z) F(z)\right|_{z=0} .
\end{aligned}
$$

Similarly, in the NCCM parametrization we have:

$$
\begin{aligned}
\bar{O} & =\left\langle\phi\left|\tilde{S}(a) e^{-S\left(a^{\dagger}\right)} O\left(a^{\dagger}, a\right) e^{S\left(a^{\dagger}\right)}\right| \phi\right\rangle \\
& =\left.\tilde{S}(d / d z) e^{-S(z)} O(z, d / d z) e^{S(z)}\right|_{z=0} .
\end{aligned}
$$

By formally expanding $O(z, d / d z)$ as an ordered multinomial in $z$ and $d / d z$, and by inserting a unit operator of the form $I=e^{S(z)} e^{-S(z)}$ between every pair of operators in this expansion, we may make use of the trivial relations:

$$
e^{-S(z)} \frac{d}{d z} e^{S(z)}=\frac{d}{d z}+S^{\prime}(z)
$$

to rewrite Eq. (44) in the equivalent NCCM form:

$$
\bar{O}=\tilde{S}(d / d z) O\left(z, d / d z+S^{\prime}(z)\right) \text {. }
$$

In principle we may also write a counterpart to Eq. (46) for the ECCM parametrization, but this is cumbersome and not very enlightening for present purposes. 


\section{Holomorphic $\mathrm{CC}$ amplitudes and the average value functional for anharmonic oscillator systems}

We now specialize to an anharmonic oscillator of order $2 K$ with $K=2,3, \ldots$, and take $V(\sqrt{2} \hat{x})=\frac{1}{2} \hat{x}^{2}+U(\sqrt{2} \hat{x})$ with $U(\sqrt{2} \hat{x})=k \hat{x}^{2 K}$. Thus:

$$
H=\frac{1}{2} \hat{p}^{2}+\frac{1}{2} \hat{x}^{2}+U(\sqrt{2} \hat{x})=\frac{1}{2}+a^{\dagger} a+k 2^{-K}\left(a^{\dagger}+a\right)^{2 K} .
$$

Due to the noncommutativity of $d / d z$ and $S^{\prime}(z)$, expressions such as in Eq. (46) for the average value of an operator are useful in the first place only for deriving low-order explicit expressions for the average value functional. Since we are now interested rather in the exact qualitative properties of the ECCM parametrization, we shall focus attention on the asymptotic analytic properties of the function $F(z)$ and the other amplitudes needed in CCM. This is done in a sufficiently reliable way by WKB methods (indeed, the NCCM parametrization for the ket state is equivalent to a WKB representation for the holomorphic wave function). For the details of such analysis we refer to Refs. $[9,10]$, and only state the most important results below.

1. All Bargmann eigenstates $F^{n}(z)$ (and thus also $\tilde{F}^{n}$ ) corresponding to Schrödinger energy eigenstates $\psi_{n}(x)$, are entire functions of order $\varrho=2$ and type $\tau=\frac{1}{2}$. Along the real axis $(z=x)$ their leading behaviour is $\propto \exp \left(-\frac{1}{2} x^{2}\right)$.

2. If the eigenfunctions are represented as in Eq. (23), each $f^{n}(z)$ is an entire function of order:

$$
v=\frac{K+1}{K}
$$

Along the imaginary axis they approach zero as:

$$
f^{n}(i y) \propto \exp \left(-\alpha_{1}|y|^{n}\right) ; \quad \alpha_{1}>0, \quad y \rightarrow \pm \infty .
$$

3. A Fourier transform of $F^{n}$ (and thus also of $\tilde{F}^{n}$ ) can be defined along the real axis:

$$
\begin{aligned}
& F_{F}^{n}(q)=\int_{-\infty}^{\infty} d x e^{-i q x} F^{n}(x) \\
& F^{n}(x)=\int_{-\infty}^{\infty} \frac{d q}{2 \pi} e^{i q x} F_{F}^{n}(q) .
\end{aligned}
$$

The function $F_{F}^{n}(q)$ is again an entire function of $q$ of order 2 and type $\frac{1}{2}$, and on the real axis it decreases as $\propto \exp \left(-\frac{1}{2} q^{2}\right)$.

4. If $F_{F}^{n}(q)$ is again written as a product:

$$
F_{F}^{n}(q)=e^{-\frac{1}{2} q^{2}} \chi_{n}(q)
$$

then the leading behaviour of $\chi_{n}(q)$ along the real axis is:

$$
\chi_{n}(q) \propto \exp \left(-\alpha_{2}|q|^{v}\right), \quad(q \rightarrow \pm \infty) .
$$

5. The function $\tilde{S}(z)$ of Eq. (38) for the ground state can be generalized for pairs of excited states:

$$
\tilde{S}^{m n}(z) \equiv \int_{-\infty}^{\infty} d x \tilde{\psi}_{m}(x) \psi_{n}\left(x-\frac{z}{\sqrt{2}}\right) \exp \left(\frac{x z}{\sqrt{2}}-\frac{z^{2}}{4}\right)
$$


All these functions are again entire functions of $z$ of order $K+1$ (as are also the Schrödinger wave functions $\psi_{n}(z)$ ), and they decrease along the real axis like $\propto \exp \left(-\alpha_{3}|x|^{K+1}\right)$. Incidentally:

$$
\tilde{S}^{m n}(0)=\delta_{m n} .
$$

In particular, $\tilde{S}^{00}$ has no zeros on the real axis.

6. The Fourier transforms of $\tilde{S}^{m n}(x)$ are:

$$
\tilde{S}_{F}^{m n}(q)=\tilde{F}_{F}^{m}(q) F^{n}(i q)
$$

For real values of $q$ they behave asymptotically as in Eq. (52), but with a different constant.

Let us now consider the average value functional for a more general state which is not an eigenstate of the Hamiltonian, but otherwise a sufficiently smooth linear combination of them with qualitatively similar properties. Starting from Eq. (43) and expressing $\tilde{F}$ through its Fourier transform we obtain:

$$
\bar{O}=\int_{-\infty}^{\infty} \frac{d q}{2 \pi} \tilde{F}_{F}(q)(O F)(i q),
$$

where $(O F)(z)=O(z, d / d z) F(z)$ is another entire function of $z$. By the properties listed above this integral is bound to converge for any reasonably well-behaved operator $O$. The NCCM form corresponding to Eq. (44) is now derived quite rigorously by observing Eq. (55):

$$
\begin{aligned}
\bar{O} & =\int_{-\infty}^{\infty} \frac{d q}{2 \pi} \tilde{S}_{F}(q) F(i q)^{-1}(O F)(i q) \\
& =\int_{-\infty}^{\infty} \frac{d q}{2 \pi} \tilde{S}_{F}(q) e^{-S(i q)} O\left(i q,-i \partial_{q}\right) e^{S(i q)} .
\end{aligned}
$$

This result could have also been written directly starting from Eq. (44). However, by proceeding in the present order one is able to prove the convergence of the above integration and to give justification to such formal expressions and manipulations as in Eqs. (32) - (34) or (44).

Since $S(z)=\ln F(z)$ and the ground-state $F^{0}(z)$ has an infinite number of zeros in the complex plane $[9,10], S(z)$ has an infinite number of logarithmic branch cut singularities. In particular, for the exact eigenstates $F^{n}(z)$ the zeros asymptotically approach the imaginary axis. Therefore, whenever the values of $S(z)$ are considered at the imaginary axis, a slight rotation is implicitly understood. In spite of this complexity it is possible to define the Fourier transform of $S(z)$ as follows:

$$
S(z)=\int_{-\infty}^{\infty} d x\left(e^{x z}-1\right) g(x)
$$

where $g(x)$ is a generalized function (i.e., a Schwartz distribution), which has a power-law singularity at the origin. It is entirely determined by the zeros of the holomorphic wave function.

Applying this representation to the definition of the amplitude $\Sigma$ from Eq. (41b), we obtain

$$
\Sigma(z)=\int_{-\infty}^{\infty} d x\left(e^{x z}-1\right) \gamma(x) ; \quad \gamma(x) \equiv \tilde{S}(x) g(x) .
$$


For the cases studied in the present paper the functions $\tilde{S}(x)$ and $\gamma(x)$ decrease very rapidly for large $|x| \rightarrow \infty$. Therefore the integral in Eq. (59) converges for all $z$ and defines $\Sigma(z)$ as an entire function.

The method applied here to represent the basic $\mathrm{CC}$ amplitudes is generalized to essentially arbitrary (but sufficiently well-behaved) systems in another article [5] in the present volume.

A convenient method to calculate the average values of arbitrary operators in terms of the coupled cluster amplitudes was introduced in Refs. [9, 10], where an appropriate generating functional was constructed. Because we are now, however, interested in the qualitative exact properties of the $\mathrm{CC}$ mappings, and assume the system to be otherwise exactly known, a different and more straightforward approach is better justified. Given the original Hamiltonian in Eq. (47) the holomorphic representation leads in the NCCM parametrization to the expression:

$$
\bar{H}=\frac{1}{2}+\int_{-\infty}^{\infty} d x \tilde{S}^{\prime}(x) x g(x)+\int_{-\infty}^{\infty} \frac{d q}{2 \pi} \tilde{S}_{F}(q) e^{-S(i q)} U\left(i q-i \partial_{q}\right) e^{S(i q)}
$$

This is given directly in terms of the NCCM amplitudes $\{S, \tilde{S}\}$, where we note that by Eq. (58) the moments of $g(x)$ are identified as the amplitudes $\left\{s_{n}\right\}$.

Our ultimate goal in the present article is to carry through the entire ECCM programme up to the point of deriving and proving the following general diagonalized $\mathrm{CC}$ expansion $[11,5]$ for states that are displaced from the stationary ground state $\left|\psi_{0}\right\rangle$ :

$$
\begin{aligned}
\bar{H}= & E_{0}+\sum_{n=1}^{\infty} \varepsilon_{n} \tilde{\psi}_{n} \psi_{n}+\sum_{k+l \geqslant 3} \frac{1}{k ! l !} \sum_{\{m\}\{n\}}\left\langle m_{1} \cdots m_{k}|\bar{H}| n_{1} \cdots n_{l}\right\rangle \\
& \times \tilde{\psi}_{m_{1}} \cdots \tilde{\psi}_{m_{k}} \psi_{n_{1}} \cdots \psi_{n_{l}},
\end{aligned}
$$

where $\varepsilon_{n}=E_{n}-E_{0}$ is the excitation energy of the $n$th excited state, and where the amplitudes $\left\{\psi_{n}, \tilde{\psi}_{n}\right\}$ are now the normal mode coordinates, and should not be confused with the previous wave functions $\psi_{n}(x)$ and $\tilde{\psi}_{n}(x)$. For this purpose the CC amplitudes (namely, either the set $\left\{s_{n}, \tilde{s}_{n}\right\}$ or the set $\left\{\sigma_{n}, \tilde{\sigma}_{n}\right\}$ ) must be expanded linearly in terms of the normal-mode coordinates $[11,5]$. This will be the subject of the next section.

\section{Expansion around the ground state}

For general infinitesimal displacements:

$$
\delta F(z)=\sum_{n=1}^{\infty} \psi_{n} F^{n}(z), \quad \delta \tilde{F}(z)=\sum_{n=1}^{\infty} \tilde{\psi}_{n} \tilde{F}^{n}(z)
$$

from the ground state the displacements of the $\mathrm{CC}$ amplitudes are uniquely and, as a matter of fact, easily calculated as functions of the sets $\left\{\psi_{n}, \tilde{\psi}_{n}\right\}$ and the known holomorphic eigenfunctions. Let us first consider the NCCM case. We obtain the expansions, accurate to first order in the amplitudes $\left\{\psi_{n}, \tilde{\psi}_{n}\right\}$ :

$$
\begin{aligned}
& S(z)=S^{0}(z)+\sum_{n=1}^{\infty} \psi_{n} S^{n}(z), \\
& \tilde{S}(z)=\tilde{S}^{00}(z)+\sum_{n=1}^{\infty}\left[\psi_{n} \tilde{S}^{0 n}(z)+\tilde{\psi}_{n} \tilde{S}^{n 0}(z)\right] .
\end{aligned}
$$


The coefficients in the Taylor expansions of $S^{n}(z), \tilde{S}^{0 n}(z)$ and $\tilde{S}^{n 0}(z)$ provide the matrix elements $\phi, \chi$ of the canonical transformation into normal modes [5]. Part of them are already defined in Eq. (53). The others are:

$$
S^{n}(z) \equiv \frac{F^{n}(z)}{F^{0}(z)}-\frac{F^{n}(0)}{F^{0}(0)} \equiv \int_{-\infty}^{\infty} d x\left(e^{x z}-1\right) g_{n}(x), \quad(n>0)
$$

where the latter equation actually defines the distribution $g_{n}(x)$, and where the corresponding ground-state relation between $S^{0}(z)$ and $g_{0}(x)$ is given by Eq. (58). By inserting these expansions into Eq. (60) for the energy and simplifying, one is able to derive the normal mode expansion of Eq. (61) for the NCCM case, namely that $\langle|\bar{H}|\rangle=E_{0} ;\langle|\bar{H}| n\rangle=\langle n|\bar{H}|\rangle=\langle|\bar{H}| n m\rangle=0 \equiv\langle m n|\bar{H}|\rangle$; $\langle m|\bar{H}| n]=\varepsilon_{n} \delta_{m n}$.

Our basic goal is now to consider arbitrary (i.e., non-infinitesimal) deviations away from equilibrium. Thus, we continue to use the parametrization of Eq. (62) but no longer restrict ourselves to $\left\{\psi_{n}, \tilde{\psi}_{n}\right\}$ being infinitesimal. The procedure is simply to insert $\mathrm{Eq}$. (62) into the average value functional for the Hamiltonian from Eq. (57). The analysis, in principle, is completely straightforward. Suffice it to say that an arbitrary matrix element in Eq. (61) can be generated by modest effort and given in the form of convergent integrals. As examples we just mention the results for the third order matrix elements, of which $\langle\operatorname{lmn}|\bar{H}|\rangle=\langle\operatorname{lm}|\bar{H}| n\rangle \equiv 0$ (since in the NCCM the expectation value is linear in the amplitudes $S$ ), and the remaining ones are:

$$
\begin{aligned}
& \langle|\bar{H}| \operatorname{lm} n\rangle^{\mathrm{NCCM}}=-\left(\varepsilon_{l}+\varepsilon_{m}+\varepsilon_{n}\right) \int_{-\infty}^{\infty} \frac{d q}{2 \pi} \tilde{F}_{F}^{0}(q) \hat{S}_{l}(i q) \hat{S}_{m}(i q) \hat{S}_{n}(i q) F^{0}(i q), \\
& \langle l|\bar{H}| m n\rangle^{\mathrm{NCCM}}=\left(\varepsilon_{l}-\varepsilon_{m}-\varepsilon_{n}\right) \int_{-\infty}^{\infty} \frac{d q}{2 \pi} \tilde{F}_{F}^{I}(q) \hat{S}_{m}(i q) \hat{S}_{n}(i q) F^{0}(i q) .
\end{aligned}
$$

In these equations

$$
\tilde{S}_{l}(z) \equiv F^{l}(z) / F^{0}(z)
$$

It is characteristic that up to third order the matrix elements can be given merely in terms of the holomorphic wave functions and the excitation energies. From fourth order, however, one obtains expressions from which the potential $U$ cannot be eliminated.

The case of the ECCM is more complicated. The basic amplitudes are first expanded as below:

$$
\begin{aligned}
& \Sigma(z)=\Sigma^{0}(z)+\sum_{n=1}^{\infty}\left[\psi_{n} \sigma_{n}^{+}(z)+\tilde{\psi}_{n} \sigma_{n}^{-}(z)\right], \\
& \tilde{\Sigma}(z)=\tilde{\Sigma}^{0}(z)+\sum_{n=1}^{\infty}\left[\psi_{n} \tilde{\sigma}_{n}^{+}(z)+\tilde{\psi}_{n} \tilde{\sigma}_{n}^{-}(z)\right],
\end{aligned}
$$

from which the coefficients of the canonical transformation can be again spelled out. We find that:

$$
\begin{aligned}
& \sigma_{n}^{+}(z)=\int_{-\infty}^{\infty} d x\left(e^{x z}-1\right)\left[\tilde{S}^{00}(x) g_{n}(x)+\tilde{S}^{0 n}(x) g_{0}(x)\right] \\
& \sigma_{n}^{-}(z)=\int_{-\infty}^{\infty} d x\left(e^{x z}-1\right) \tilde{S}^{n 0}(x) g_{0}(x) .
\end{aligned}
$$


where the functions $g_{n}(x)$ are as defined by Eq. (63). The other coefficients are:

$$
\tilde{\sigma}_{n}^{+}(z)=\frac{\tilde{S}^{0 n}(z)}{\tilde{S}^{00}(z)} ; \quad \tilde{\sigma}_{n}^{-}(z)=\frac{\tilde{S}^{n 0}(z)}{\tilde{S}^{00}(z)} .
$$

To proceed further one has to express the NCCM amplitudes $\tilde{S}(x), \tilde{S}_{F}(q)$, $g(x)$ and $S(z)$, appearing in Eq. (60) for the energy, in terms of the ECCM amplitudes in Eq. (66). The connection is nonlinear. We obtain:

$$
\widetilde{S}(x)=\tilde{S}^{00}(x) \exp \left\{\sum_{n=1}^{\infty}\left[\psi_{n} \tilde{\sigma}_{n}^{+}(x)+\tilde{\psi}_{n} \tilde{\sigma}_{n}^{-}(x)\right]\right\}
$$

and the higher-order terms in the expansion of $\tilde{S}_{F}(q)$ are obviously rather complicated integrals. Also:

$$
g(x)=g_{0}(x)(1+Z) e^{-Z}+e^{-Z} \sum_{n=1}^{\infty} \psi_{n} g_{n}(x),
$$

where $Z \equiv \delta \tilde{\Sigma}(x)=\sum_{n=1}^{\infty}\left(\psi_{n} \tilde{\sigma}_{n}^{+}+\tilde{\psi}_{n} \tilde{\sigma}_{n}^{-}\right)$. From these equations and the definition of Eq. (58), we obtain $S(z)$ as a nonlinear expansion of the coordinates $\left\{\psi_{n}, \tilde{\psi}_{n}\right\}$.

The evaluation of the individual matrix elements of the ECCM Hamiltonian is now quite laborious. Nevertheless, the lowest-order matrix elements up to third order are again rather straightforward. The results are:

$$
\begin{aligned}
\langle l m n|\bar{H}|\rangle^{\mathrm{ECCM}}= & -\left(\varepsilon_{l}+\varepsilon_{m}+\varepsilon_{n}\right) \int_{-\infty}^{\infty} d x \tilde{S}^{00}(x) g_{0}(x) \tilde{\sigma}_{l}^{-}(x) \tilde{\sigma}_{m}^{-}(x) \tilde{\sigma}_{n}^{-}(x) \\
\langle\operatorname{lm}|\bar{H}| n\rangle^{\mathrm{ECCM}}= & \left(\varepsilon_{n}-\varepsilon_{l}-\varepsilon_{m}\right) \int_{-\infty}^{\infty} d x \tilde{S}^{00}(x) \tilde{\sigma}_{l}^{-}(x) \tilde{\sigma}_{m}^{-}(x)\left[g_{0}(x) \tilde{\sigma}_{n}^{+}(x)+g_{n}(x)\right] \\
\langle l|\bar{H}| m n\rangle^{\mathrm{ECCM}}= & \langle l|\bar{H}| m n\rangle^{\mathrm{NCCM}}+\left(\varepsilon_{m}+\varepsilon_{n}-\varepsilon_{l}\right) \int_{-\infty}^{\infty} d x \tilde{S}^{00}(x) \tilde{\sigma}_{l}^{-}(x) \\
& \times\left[g_{0}(x) \tilde{\sigma}_{m}^{+}(x) \tilde{\sigma}_{n}^{+}(x)+g_{m}(x) \tilde{\sigma}_{n}^{+}(x)+g_{n}(x) \tilde{\sigma}_{m}^{+}(x)\right] \\
\langle|\bar{H}| l m n\rangle^{\mathrm{ECCM}}= & \langle|\bar{H}| l m n\rangle^{\mathrm{NCCM}}+\left(\varepsilon_{l}+\varepsilon_{m}+\varepsilon_{n}\right) \int_{-\infty}^{\infty} d x \tilde{S}^{00}(x) \\
& \times\left[g_{0}(x) \tilde{\sigma}_{l}^{+}(x) \tilde{\sigma}_{m}^{+}(x) \tilde{\sigma}_{n}^{+}(x)+g_{l}(x) \tilde{\sigma}_{m}^{+}(x) \tilde{\sigma}_{n}^{+}(x)\right. \\
& \left.+g_{m}(x) \tilde{\sigma}_{l}^{+}(x) \tilde{\sigma}_{n}^{+}(x)+g_{n}(x) \tilde{\sigma}_{l}^{+}(x) \tilde{\sigma}_{m}^{+}(x)\right]
\end{aligned}
$$

Higher-order matrix elements are more complicated to determine. Nevertheless it is evident that any desired coefficient can be evaluated with finite effort. All functions appearing in the above expressions are qualitatively well known, and all integrals can be readily proven to converge. Thus we have been able to carry through the $\mathrm{CC}$ programmes up to the point of deriving the diagonalized mapped classical NCCM and ECCM Hamiltonians. However, because of the drastic simplicity of the model field theory in the present case, the derived expansions are otherwise not intrinsically very interesting. The expansions 
derived in this Section around the ground state are not yet sufficient to unravel the global structure of the NCCM or ECCM phase spaces. Although it would take us too far afield to attempt an explicit construction in this paper, it is worth mentioning that the method of diagonalizing the second-order displacements of the classical $\bar{H}$ can be applied around an essentially arbitrary point in the Hilbert space. The geometrical connections between the local parametrizations for neighbouring points would shed further light into this problem and allow a more careful study of such concepts as the phase portraits or energy surfaces in the CC phase space, for example. We believe that the most significant aspect of the present problem is the very existence of the $\mathrm{CC}$ phase spaces, a fact that has not so far been proven for any Hilbert space of infinite dimensionality, and the possible promises in implementing the methods developed here for other more realistic problems in field theory and many-body theory.

\section{Summary}

We have seen in the previous history of the coupled cluster methods a wealth of successful applications to many-body problems in both physics and chemistry as well as to problems in field theories. The practical complexity of all such applications to realistic systems has diverted interest away from the fundamental mathematical concepts towards more practical computational aspects. It is clear that for finite Hilbert spaces the $\mathrm{CC}$ amplitudes and thus the $\mathrm{CC}$ phase spaces definitely exist. To our knowledge the present investigation (together with Ref. [10]) is the first to prove rigorously the existence of the NCCM and ECCM phase spaces for a nontrivial infinite Hilbert space.

In conclusion, it is clear that the holomorphic representation is a very powerful tool in the consideration of rather formal questions of fundamental importance. It may also be of much more practical use in actual physical or chemical applications. For example, another feature that we have not stressed in the present paper, but which should be apparent to the reader, is that the holomorphic representation also enables us to provide a completely algebraic description of all aspects of all three of the independent-cluster parametrizations, namely those of the CIM, NCCM and ECCM. One obvious example of this algebraization is the topological connectivity properties of the various terms or diagrams that each parametrization provides for the expectation values of the Hamiltonian and other operators. In particular, the specific linked and doublelinked properties of the NCCM and ECCM diagrams respectively [3-5] can be put onto a completely rigorous algebraic footing. The existence of such techniques, which we have more fully explored elsewhere $[9,10]$, may also be of practical significance in later applications to realistic systems.

Acknowledgement. One of us (R.F.B.) gratefully acknowledges the support of a research grant from the Science and Engineering Research Council (SERC) of Great Britain.

\section{References}

1. Bartlett RJ (1991) Theor Chim Acta 80

2. Bishop RF (1991) Theor Chim Acta 80

3. Arponen J (1983) Ann Phys (NY) 151:311 
4. Arponen JS, Bishop RF, Pajanne E (1987) Phys Rev A 36:2519

5. Arponen JS (1991) Theor Chim Acta 80

6. Bargmann V (1961) Comm Pure Appl Math 14:180, 187; ibid (1967) idem 20:1; Bargmann V, Butera P, Girardello L, Klauder JR (1971) Rep Math Phys 2:221

7. Bender CM, Wu TT (1969) Phys Rev 184:1231; ibid (1971) Phys Rev Lett 27:461; ibid (1973) Phys Rev D 7:1620; Simon B (1970) Ann Phys (NY) 58:76, Truong TT (1975) J Math Phys 16:1034; Balian R, Parisi G, Voros A (1978) Phys Rev Lett 41:1141; Hioe FT, MacMillen D, Montroll EW (1978) Phys Rep 43:305; Voros A (1983) Ann Inst Henri Poincaré A39:211

8. Hsue CS, Chern JL (1984) Phys Rev D 29:643; Kaulfuss UB, Altenbokum M (1986) Phys Rev D 33:3658; Kümmel HG (1988) The anharmonic oscillator revisited. In: Arponen JS, Bishop RF, Manninen M (eds) Condensed matter theories, vol. 3. Plenum, New York, p 21: Bishop RF, Flynn MF (1988) Phys Rev A 38:2211; Bishop RF, Boscá MC, Flynn MF (1988) Phys Lett A132:440; Aalto E, Arponen JS, Bishop RF (1990) On the Bargmann space approach to the extended coupled cluster method for simple anharmonic systems. In: Aguilera-Navarro VC (ed) Condensed matter theories, vol 5, Plenum, New York, p 297

9. Arponen JS, Bishop RF (1990) Phys Rev Lett 64:111

10. Arponen JS, Bishop RF (1991) Ann Phys (NY) 207:171

11. Arponen JS, Bishop RF, Pajanne E (1987) Phys Rev A36:2539 